DOI: $10.15393 /$ j3.art.2019.5150

UDC 517.521

R. M. GADZhimirzaeV

\title{
SOBOLEV-ORTHONORMAL SYSTEM OF FUNCTIONS GENERATED BY THE SYSTEM OF LAGUERRE FUNCTIONS
}

\begin{abstract}
We consider the system of functions $\lambda_{r, n}^{\alpha}(x)(r \in \mathbb{N}$, $n=0,1,2, \ldots)$, orthonormal with respect to the Sobolev-type inner product $\langle f, g\rangle=\sum_{\nu=0}^{r-1} f^{(\nu)}(0) g^{(\nu)}(0)+\int_{0}^{\infty} f^{(r)}(x) g^{(r)}(x) d x$ and generated by the orthonormal Laguerre functions. The Fourier series in the system $\left\{\lambda_{r, n}^{\alpha}(x)\right\}_{k=0}^{\infty}$ is shown to uniformly converge to the function $f \in W_{L^{p}}^{r}$ for $\frac{4}{3}<p<4, \alpha \geqslant 0, x \in[0, A]$, $0 \leqslant A<\infty$. Recurrence relations are obtained for the system of functions $\lambda_{r, n}^{\alpha}(x)$. Moreover, we study the asymptotic properties of the functions $\lambda_{1, n}^{\alpha}(x)$ as $n \rightarrow \infty$ for $0 \leqslant x \leqslant \omega$, where $\omega$ is a fixed positive real number.
\end{abstract}

Key words: Laguerre polynomials, Laguerre functions, inner product of Sobolev type, Sobolev-orthonormal functions, recurrence relations, Fourier series, asymptotic formula

2010 Mathematical Subject Classification: 42C10, 65Q30

\section{Introduction.}

Let $L^{p}$ be the space of measurable functions $f$ defined on the semiaxis $[0, \infty)$, such that

$$
\|f\|_{L^{p}}=\left(\int_{0}^{\infty}|f(x)|^{p} d x\right)^{\frac{1}{p}}<\infty, 1 \leqslant p<\infty,
$$

$W_{L^{p}}^{r}$ be the space of $r-1$ times continuously differentiable functions $f$ for which $f^{(r-1)}$ is absolutely continuous on an arbitrary segment $[a, b] \subset[0, \infty)$ and $f^{(r)} \in L^{p}$. By $\lambda_{n}^{\alpha}(x)(n=0,1, \ldots)$ we denote the Laguerre function defined by the formula

$$
\lambda_{n}^{\alpha}(x)=\sqrt{\rho(x)} l_{n}^{\alpha}(x),
$$

(c) Petrozavodsk State University, 2019 
where $\rho(x)=e^{-x} x^{\alpha}, l_{n}^{\alpha}(x)$ is the orthonormal Laguerre polynomial (13). It is well known that for $\alpha>-1$ the system of functions $\left\{\lambda_{n}^{\alpha}(x)\right\}_{n=0}^{\infty}$ is orthonormal with respect to the inner product

$$
\left\langle\lambda_{m}^{\alpha}, \lambda_{n}^{\alpha}\right\rangle=\int_{0}^{\infty} \lambda_{m}^{\alpha}(x) \lambda_{n}^{\alpha}(x) d x .
$$

The system of Laguerre functions $\left\{\lambda_{n}^{\alpha}(x)\right\}_{n=0}^{\infty}$ generates on $[0, \infty)$ a system of functions $\lambda_{r, n}^{\alpha}(x)(r \in \mathbb{N}, n=0,1, \ldots)$ orthonormal for $\alpha>-1$ with respect to the Sobolev type inner product

$$
\langle f, g\rangle=\sum_{\nu=0}^{r-1} f^{(\nu)}(0) g^{(\nu)}(0)+\int_{0}^{\infty} f^{(r)}(x) g^{(r)}(x) d x .
$$

The functions $\lambda_{r, n}^{\alpha}(x)$ are defined by means of equalities (15) and (16). In this paper, we show that the Fourier series in the system $\left\{\lambda_{r, n}^{\alpha}(x)\right\}_{k=0}^{\infty}$ converges uniformly to the function $f \in W_{L^{p}}^{r}$ for $\alpha \geqslant 0, \frac{4}{3}<p<4$, $x \in[0, A], 0 \leqslant A<\infty$. Recurrence relations are obtained for the system of functions $\lambda_{r, n}^{\alpha}(x)$ and can be used for calculating the values of $\lambda_{r, n}^{\alpha}(x)$ for any $x$ and $n$. Moreover, we study the asymptotic properties of the functions $\lambda_{1, n}^{\alpha}(x)$ as $n \rightarrow \infty$ for $0 \leqslant x \leqslant \omega$, where $\omega$ is a fixed positive real number. Using these asymptotic properties, we obtained estimates for the functions $\lambda_{1, n}^{\alpha}(x)$ on the interval $[0, \omega]$.

\section{Some information on the Laguerre polynomials and La- guerre functions.}

To study Sobolev-orthonormal functions generated by Laguerre functions, we need some properties of the Laguerre polynomials and Laguerre functions that are given in this section.

Let $\alpha$ be an arbitrary real number. Then for the Laguerre polynomials we have [12]:

- The Rodrigues formula

$$
L_{n}^{\alpha}(x)=\frac{1}{n !} x^{-\alpha} e^{x}\left\{x^{n+\alpha} e^{-x}\right\}^{(n)} .
$$

- The orthogonality relations

$$
\int_{0}^{\infty} L_{n}^{\alpha}(x) L_{m}^{\alpha}(x) \rho(x) d x=\delta_{n, m} h_{n}^{\alpha} \quad(\alpha>-1),
$$


where $\rho(x)=e^{-x} x^{\alpha}, \delta_{n, m}$ is the Kronecker symbol, $h_{n}^{\alpha}=\left(\begin{array}{c}n+\alpha \\ n\end{array}\right) \Gamma(\alpha+1)$.

- The equalities

$$
\begin{gathered}
\frac{d}{d x} L_{n}^{\alpha}(x)=-L_{n-1}^{\alpha+1}(x) . \\
L_{n}^{-k}(x)=\frac{(-x)^{k}}{n^{[k]}} L_{n-k}^{k}(x),
\end{gathered}
$$

where $k$ is a positive integer number and $1 \leqslant k \leqslant n, n^{[0]}=1$, $n^{[k]}=n(n-1) \cdots(n-k+1)$.

$$
x L_{n}^{\alpha+1}(x)=(n+\alpha+1) L_{n}^{\alpha}(x)-(n+1) L_{n+1}^{\alpha}(x) ;
$$

- The recurrence formula

$$
\begin{gathered}
L_{0}^{\alpha}(x)=1, \quad L_{1}^{\alpha}(x)=-x+\alpha+1, \\
n L_{n}^{\alpha}(x)=(-x+2 n+\alpha-1) L_{n-1}^{\alpha}(x)-(n+\alpha-1) L_{n-2}^{\alpha}(x), n=2,3, \ldots
\end{gathered}
$$

- Theorem. [12, p.199, Theorem 8.22.4] For $\alpha>-1$, we have

$$
\begin{gathered}
e^{-\frac{x}{2}} x^{\frac{\alpha}{2}} L_{n}^{\alpha}(x)=N^{-\frac{\alpha}{2}} \frac{\Gamma(n+\alpha+1)}{n !} J_{\alpha}\left(2(N x)^{\frac{1}{2}}\right)+O\left(n^{\frac{\alpha}{2}-\frac{3}{4}}\right), \\
N=n+\frac{\alpha+1}{2}, x>0,
\end{gathered}
$$

the bound holding uniformly in $0<x \leqslant \omega$ ( $\omega$ is a fixed positive number). More precisely, the following bounds are valid:

$$
\left.\begin{array}{c}
x^{\frac{5}{4}} O\left(n^{\frac{\alpha}{2}-\frac{3}{4}}\right), \frac{c}{n} \leqslant x \leqslant \omega, \\
x^{\frac{\alpha}{2}+2} O\left(n^{\alpha}\right), 0<x \leqslant \frac{c}{n}
\end{array}\right\} .
$$

In $(7), J_{\alpha}(x)$ is the Bessel function of the first kind; for it the following asymptotic formula holds [12, p.15, formula 1.71.7]:

$$
J_{\alpha}(x)=\left(\frac{2}{\pi x}\right)^{\frac{1}{2}} \cos \left(x-\frac{\alpha \pi}{2}-\frac{\pi}{4}\right)+O\left(x^{-\frac{3}{2}}\right), x \rightarrow+\infty ;
$$

- The weight estimate $[1,4]$

$$
e^{-\frac{x}{2}}\left|L_{n}^{\alpha}(x)\right| \leqslant c(\alpha) A_{n}^{\alpha}(x), \alpha>-1 .
$$


Here and henceforth, $c$ and $c(\alpha)$ are positive real numbers depending only on the indicated parameters,

$$
A_{n}^{\alpha}(x)= \begin{cases}\theta_{n}^{\alpha}, & 0 \leqslant x \leqslant \frac{1}{\theta_{n}}, \\ \theta_{n}^{\frac{\alpha}{2}-\frac{1}{4}} x^{-\frac{\alpha}{2}-\frac{1}{4}}, & \frac{1}{\theta_{n}}<x \leqslant \frac{\theta_{n}}{2}, \\ {\left[\theta_{n}\left(\theta_{n}^{\frac{1}{3}}+\left|x-\theta_{n}\right|\right)\right]^{-\frac{1}{4}},} & \frac{\theta_{n}}{2}<x \leqslant \frac{3 \theta_{n}}{2}, \\ e^{-\frac{x}{4}}, & \frac{3 \theta_{n}}{2}<x,\end{cases}
$$

where $\theta_{n}=\theta_{n}(\alpha)=4 n+2 \alpha+2$.

- The differentiation formula [2, p.191, formula 27]

$$
\left[x^{\alpha} L_{n}^{\alpha}(x)\right]^{(m)}=(n-m+\alpha+1)_{m} x^{\alpha-m} L_{n}^{\alpha-m}(x),
$$

where $(n)_{0}=1,(n)_{m}=n(n+1) \cdots(n+m-1), m \geqslant 1$.

It follows from (3) that the corresponding orthonormal system of the Laguerre polynomials has the form:

$$
l_{n}^{\alpha}(x)=\left(h_{n}^{\alpha}\right)^{-\frac{1}{2}} L_{n}^{\alpha}(x), \quad n=0,1, \ldots,
$$

so

$$
\int_{0}^{\infty} l_{n}^{\alpha}(x) l_{m}^{\alpha}(x) \rho(x) d x=\delta_{n, m} \quad(\alpha>-1) .
$$

From (6) and (13), we immediately obtain a recurrence formula for $l_{n}^{\alpha}(x)$ :

$$
\left.\begin{array}{c}
l_{0}^{\alpha}(x)=\frac{1}{\sqrt{\Gamma(\alpha+1)}}, \quad l_{1}^{\alpha}(x)=\frac{-x+\alpha+1}{\sqrt{\Gamma(\alpha+2)}}, \\
l_{n}^{\alpha}(x)=\left(a_{n}-b_{n} x\right) l_{n-1}^{\alpha}(x)-c_{n} l_{n-2}^{\alpha}(x), \quad n=2,3, \ldots
\end{array}\right\},
$$

where

$$
\begin{gathered}
a_{n}=a_{n}(\alpha)=\frac{2 n+\alpha-1}{[n(n+\alpha)]^{\frac{1}{2}}}, \quad b_{n}=b_{n}(\alpha)=\frac{1}{[n(n+\alpha)]^{\frac{1}{2}}}, \\
c_{n}=c_{n}(\alpha)=\left[\frac{(n-1)(n+\alpha-1)}{n(n+\alpha)}\right]^{\frac{1}{2}} .
\end{gathered}
$$


A similar recurrence formula holds for the functions $\lambda_{n}^{\alpha}(x)$ :

$$
\left.\begin{array}{c}
\lambda_{0}^{\alpha}(x)=\frac{\sqrt{\rho(x)}}{\sqrt{\Gamma(\alpha+1)}}, \quad \lambda_{1}^{\alpha}(x)=\frac{\sqrt{\rho(x)}(-x+\alpha+1)}{\sqrt{\Gamma(\alpha+2)}}, \\
\lambda_{n}^{\alpha}(x)=\left(a_{n}-b_{n} x\right) \lambda_{n-1}^{\alpha}(x)-c_{n} \lambda_{n-2}^{\alpha}(x), \quad n=2,3, \ldots
\end{array}\right\} .
$$

In the sequel, we need the following property of the functions $\lambda_{n}^{\alpha}(x)$. Theorem A. [1, Theorem 1] Let $f \in L^{p}, \frac{4}{3}<p<4, \alpha \geqslant 0$. Define $a_{n}=\int_{0}^{\infty} \lambda_{n}^{\alpha}(x) f(x) d x$ and set $S_{n}(x)=\sum_{k=0}^{n} a_{k} \lambda_{k}^{\alpha}(x)$. Then $\left\|S_{n}-f\right\|_{L^{p}} \rightarrow 0$ as $n \stackrel{0}{\rightarrow} \infty$.

3. On the Sobolev orthonormal functions generated by the Laguerre functions.

Definition 1. For a given $r \in \mathbb{N}$, define the functions $\lambda_{r, n}^{\alpha}(x)$, $n=0,1, \ldots$, by

$$
\begin{gathered}
\lambda_{r, r+n}^{\alpha}(x)=\frac{1}{(r-1) !} \int_{0}^{x}(x-t)^{r-1} \lambda_{n}^{\alpha}(t) d t, \quad n=0,1, \ldots \\
\lambda_{r, n}^{\alpha}(x)=\frac{x^{n}}{n !}, \quad n=0,1, \ldots, r-1 .
\end{gathered}
$$

Consider the problem of computing the functions $\lambda_{r, r+n}^{\alpha}(x)$ for any $n$ and $x$. Note that $\lambda_{0, n}^{\alpha}(x)=\lambda_{n}^{\alpha}(x), \lambda_{1,0}^{\alpha}(x)=1, \lambda_{1,1}^{\alpha}(x)=\int_{0}^{x} \lambda_{0}^{\alpha}(t) d t$ by definition.

Theorem 1. Let $\alpha>-1$. Then the following recurrence relations hold:

$$
\begin{gathered}
\lambda_{r, n}^{\alpha}(x)=\frac{x}{n} \lambda_{r, n-1}^{\alpha}(x), \quad 1 \leqslant n \leqslant r-1 \\
r \lambda_{r+1, r+1}^{\alpha}(x)=(x-2 r-\alpha) \lambda_{r, r}^{\alpha}(x)+2 x \lambda_{r-1, r-1}^{\alpha}(x), r \geqslant 1 ; \\
\sqrt{(n+1)(n+\alpha+1)} \lambda_{1, n+2}^{\alpha}(x)=2 x \lambda_{n}^{\alpha}(x)-\lambda_{1, n+1}^{\alpha}(x)+ \\
+\sqrt{n(n+\alpha)} \lambda_{1, n}^{\alpha}(x), \quad n \geqslant 1 ; \\
r \lambda_{r+1, r+n}^{\alpha}(x)=\sqrt{n(n+\alpha)} \lambda_{r, r+n}^{\alpha}(x)+(x-2 n-\alpha+1) \lambda_{r, r+n-1}^{\alpha}(x)+ \\
+\sqrt{(n-1)(n+\alpha-1)} \lambda_{r, r+n-2}^{\alpha}(x), r \geqslant 1, \quad n=2,3, \ldots
\end{gathered}
$$


Proof. The equality (17) is obvious. Let us prove the relation (18). From the definition of the functions $\lambda_{r, r+n}^{\alpha}(x)$ and integrating by parts, we have:

$$
\begin{aligned}
\lambda_{r, r}^{\alpha}(x)= & \frac{1}{(r-1) !} \int_{0}^{x}(x-t)^{r-1} \lambda_{0}^{\alpha}(t) d t= \\
= & \frac{1}{\sqrt{\Gamma(\alpha+1)}} \frac{1}{(r-1) !} \int_{0}^{x}(x-t)^{r-1} e^{-\frac{t}{2}} t^{\frac{\alpha}{2}} d t= \\
= & \frac{2}{(\alpha+2) \sqrt{\Gamma(\alpha+1)}} \frac{1}{(r-1) !} \int_{0}^{x}(x-t)^{r-1} e^{-\frac{t}{2}} d\left(t^{\frac{\alpha}{2}+1}\right)= \\
= & -\frac{2}{\alpha+2} \frac{1}{(r-2) !} \int_{0}^{x}(x-t)^{r-2}(x-t-x) \lambda_{0}^{\alpha}(t) d t- \\
& -\frac{1}{\alpha+2} \frac{1}{(r-1) !} \int_{0}^{x}(x-t)^{r-1}(x-t-x) \lambda_{0}^{\alpha}(t) d t= \\
= & -\frac{2(r-1)}{\alpha+2} \lambda_{r, r}^{\alpha}(x)+\frac{2}{\alpha+2} x \lambda_{r-1, r-1}^{\alpha}(x)-\frac{r}{\alpha+2} \lambda_{r+1, r+1}^{\alpha}(x)+ \\
& +\frac{1}{\alpha+2} x \lambda_{r, r}^{\alpha}(x) .
\end{aligned}
$$

Hence, we obtain (18). We now establish the equality (19):

$$
\begin{aligned}
\lambda_{1, n+1}^{\alpha}(x) & =\int_{0}^{x} \lambda_{n}^{\alpha}(t) d t=\frac{2}{\alpha+2} \int_{0}^{x} e^{-\frac{t}{2}} l_{n}^{\alpha}(t) d\left(t^{\frac{\alpha}{2}+1}\right)=\frac{2}{\alpha+2} x \lambda_{n}^{\alpha}(x)+ \\
& +\frac{1}{\alpha+2} \int_{0}^{x} e^{-\frac{t}{2}} t^{\frac{\alpha}{2}+1} l_{n}^{\alpha}(t) d t-\frac{2}{\alpha+2} \int_{0}^{x} e^{-\frac{t}{2}} t^{\frac{\alpha}{2}+1}\left(l_{n}^{\alpha}(t)\right)^{\prime} d t .
\end{aligned}
$$

Consider separately the second and the third terms of the right-hand side of the last equality. From (14) we have:

$$
\begin{gathered}
\int_{0}^{x} e^{-\frac{t}{2}} t^{\frac{\alpha}{2}+1} l_{n}^{\alpha}(t) d t=\int_{0}^{x} t \lambda_{n}^{\alpha}(t) d t=\int_{0}^{x}\left[-\sqrt{(n+1)(n+\alpha+1)} \lambda_{n+1}^{\alpha}(t)+\right. \\
\left.+(2 n+\alpha+1) \lambda_{n}^{\alpha}(t)-\sqrt{n(n+\alpha)} \lambda_{n-1}^{\alpha}(t)\right] d t=
\end{gathered}
$$




$$
=-\sqrt{(n+1)(n+\alpha+1)} \lambda_{1, n+2}^{\alpha}(x)+(2 n+\alpha+1) \lambda_{1, n+1}^{\alpha}(x)-\sqrt{n(n+\alpha)} \lambda_{1, n}^{\alpha}(x) .
$$

Further, from the equalities (4), (5), and (13) it follows that

$$
\begin{gathered}
\left(l_{n}^{\alpha}(t)\right)^{\prime}=-\sqrt{n} l_{n-1}^{\alpha+1}(t), \\
t l_{n-1}^{\alpha+1}(t)=\sqrt{n+\alpha} l_{n-1}^{\alpha}(t)-\sqrt{n} l_{n}^{\alpha}(t) .
\end{gathered}
$$

Then

$$
\begin{gathered}
\int_{0}^{x} e^{-\frac{t}{2} t^{\frac{\alpha}{2}+1}\left(l_{n}^{\alpha}(t)\right)^{\prime} d t}=-\sqrt{n} \int_{0}^{x} e^{-\frac{t}{2}} t^{\frac{\alpha}{2}} t l_{n-1}^{\alpha+1}(t) d t= \\
=-\sqrt{n} \int_{0}^{x} e^{-\frac{t}{2} t^{\frac{\alpha}{2}}}\left[\sqrt{n+\alpha} l_{n-1}^{\alpha}(t)-\sqrt{n} l_{n}^{\alpha}(t)\right] d t= \\
=-\sqrt{n(n+\alpha)} \lambda_{1, n}^{\alpha}(x)+n \lambda_{1, n+1}^{\alpha}(x) .
\end{gathered}
$$

From (22), (23) and (21) we obtain (19).

Let us proceed to the proof of (20). By definition,

$$
\lambda_{r, r+n}^{\alpha}(x)=\frac{1}{(r-1) !} \int_{0}^{x}(x-t)^{r-1} \lambda_{n}^{\alpha}(t) d t .
$$

Replace the function $\lambda_{n}^{\alpha}(t)$ by the right-hand side of the equality (14):

$$
\begin{aligned}
& \lambda_{r, r+n}^{\alpha}(x)=\frac{1}{(r-1) !} \int_{0}^{x}(x-t)^{r-1}\left[\left(a_{n}-b_{n} t\right) \lambda_{n-1}^{\alpha}(t)-c_{n} \lambda_{n-2}^{\alpha}(t)\right] d t= \\
& =a_{n} \lambda_{r, r+n-1}^{\alpha}(x)-\frac{b_{n}}{(r-1) !} \int_{0}^{x}(x-t)^{r-1} t \lambda_{n-1}^{\alpha}(t) d t-c_{n} \lambda_{r, r+n-2}^{\alpha}(x)= \\
& =a_{n} \lambda_{r, r+n-1}^{\alpha}(x)+\frac{b_{n}}{(r-1) !} \int_{0}^{x}(x-t)^{r-1}(x-t-x) \lambda_{n-1}^{\alpha}(t) d t-c_{n} \lambda_{r, r+n-2}^{\alpha}(x)= \\
& =a_{n} \lambda_{r, r+n-1}^{\alpha}(x)+b_{n} r \lambda_{r+1, r+n}^{\alpha}(x)-b_{n} x \lambda_{r, r+n-1}^{\alpha}(x)-c_{n} \lambda_{r, r+n-2}^{\alpha}(x) . \quad(24)
\end{aligned}
$$

Now divide both sides of (24) by $b_{n}$ and obtain the relation (20). 
Remark 1. Formula (19) is also valid for $n=0$.

Note that the systems defined by means of formulae (15), (16) in the general case, when an arbitrary orthonormal system $\varphi_{k}(x)(k=0,1, \ldots)$ is used as the generating system, were considered in the works [5-10]. In particular, in the paper [5] the following theorem was proved.

Theorem B. Assume that the functions $\varphi_{k}(x)(k=0,1, \ldots)$ form a complete in $L_{\rho}^{2}(a, b)$ orthonormal system with respect to the weight $\rho(x)$ on the interval $[a, b]$. Then the system $\left\{\varphi_{r, k}(x)\right\}_{k=0}^{\infty}$, generated by the $\left\{\varphi_{k}(x)\right\}_{k=0}^{\infty}$ by means of

$$
\begin{gathered}
\varphi_{r, r+k}(x)=\frac{1}{(r-1) !} \int_{a}^{x}(x-t)^{r-1} \varphi_{k}(t) d t, \quad k=0,1, \ldots \\
\varphi_{r, k}(x)=\frac{(x-a)^{k}}{k !}, \quad k=0,1, \ldots, r-1,
\end{gathered}
$$

is complete in $W_{L_{\rho}^{2}(a, b)}^{r}$ and orthonormal with respect to the inner product

$$
\langle f, g\rangle=\sum_{\nu=0}^{r-1} f^{(\nu)}(a) g^{(\nu)}(a)+\int_{a}^{b} f^{(r)}(t) g^{(r)}(t) \rho(t) d t .
$$

Note that Theorem B holds for infinite intervals too. The following statement is immediately deduced from Theorem B.

Corollary 1. If $\alpha>-1$, then the system of functions $\lambda_{r, n}^{\alpha}(x)$, generated by the Laguerre functions $\lambda_{n}^{\alpha}(x)$ by means of equalities (15) and (16), is complete in $W_{L^{2}}^{r}$ and orthonormal with respect to the inner product (2).

Further, from (15), (16), and the integrand differentiation formula [3, sec. 509, p. 667] for almost all $x \in[0, \infty)$ we have

$$
\left(\lambda_{r, k}^{\alpha}(x)\right)^{(\nu)}= \begin{cases}\lambda_{r-\nu, k-\nu}^{\alpha}(x), & 0 \leqslant \nu \leqslant r-1, r \leqslant k \\ \lambda_{k-r}^{\alpha}(x), & \nu=r \leqslant k \\ \lambda_{r-\nu, k-\nu}^{\alpha}(x), & \nu \leqslant k<r \\ 0, & k<\nu \leqslant r\end{cases}
$$

where $\lambda_{0, n}^{\alpha}(x)=\lambda_{n}^{\alpha}(x)$ by convention. 
It is easily seen from (2), (15)-(25) that the Fourier series of the function $f \in W_{L^{2}}^{r}$ in the system $\left\{\lambda_{r, k}^{\alpha}(x)\right\}_{k=0}^{\infty}$

$$
f(x) \sim \sum_{k=0}^{\infty} c_{r, k}^{\alpha}(f) \lambda_{r, k}^{\alpha}(x)
$$

has the following form:

$$
f(x) \sim \sum_{k=0}^{r-1} f^{(k)}(0) \frac{x^{k}}{k !}+\sum_{k=r}^{\infty} c_{r, k}^{\alpha}(f) \lambda_{r, k}^{\alpha}(x),
$$

where

$$
c_{r, k}^{\alpha}(f)=\int_{0}^{\infty} f^{(r)}(t) \lambda_{k-r}^{\alpha}(t) d t, \quad k=r, r+1, \ldots
$$

Note that the Fourier series (26) can be defined for any function $f \in W_{L^{p}}^{r}$, $p \geqslant 1$. To this end, we show the existence of the coefficients $c_{r, k}^{\alpha}(f)$ defined by the equality (27). Using the Hölder inequality, we have

$$
\begin{aligned}
\left|c_{r, k}^{\alpha}(f)\right| \leqslant\left(\int_{0}^{\infty}\left|f^{(r)}(t)\right|^{p} d t\right)^{\frac{1}{p}}\left(\int_{0}^{\infty}\left|\lambda_{k-r}^{\alpha}(t)\right|^{q} d t\right)^{\frac{1}{q}} \leqslant & \\
& \leqslant M\left\|f^{(r)}\right\|_{L^{p}}, k=r, r+1, \ldots,
\end{aligned}
$$

where $M$ is a positive real number and $1 / p+1 / q=1$. Consider the problem of uniform convergence of the Fourier series (26) to the function $f \in W_{L^{p}}^{r}$. To prove the following theorem, we use the same technique as in [11].

Theorem 2. Let $\alpha \geqslant 0,0 \leqslant A<\infty, \frac{4}{3}<p<4, f \in W_{L^{p}}^{r}$. Then the series (26) converges uniformly on $[0, A]$ to the function $f$.

Proof. Since $f \in W_{L^{p}}^{r}$, then, first, $f^{(r)} \in L^{p}$, and, therefore, in the metric of the space $L^{p}$ we have (see Theorem A)

$$
\begin{gathered}
f^{(r)}(x)=\sum_{k=0}^{\infty} c_{r, k}^{\alpha}\left(f^{(r)}\right) \lambda_{k}^{\alpha}(x), \\
c_{r, k}^{\alpha}\left(f^{(r)}\right)=\int_{0}^{\infty} f^{(r)}(t) \lambda_{k}^{\alpha}(t) d t, k=0,1, \ldots
\end{gathered}
$$


Second, we can write the Taylor formula for the function $f$, with the remainder in the integral form:

$$
f(x)=\sum_{k=0}^{r-1} f^{(k)}(0) \frac{x^{k}}{k !}+\frac{1}{(r-1) !} \int_{0}^{x}(x-t)^{r-1} f^{(r)}(t) d t .
$$

Further, denote by $S_{r, n}^{\alpha}(f, x)$ and $S_{n}^{\alpha}\left(f^{(r)}, x\right)$ the partial sums of the series (26) and (28), respectively:

$$
\begin{gathered}
S_{r, n}^{\alpha}(f, x)=\sum_{k=0}^{r-1} f^{(k)}(0) \frac{x^{k}}{k !}+\sum_{k=r}^{n} c_{r, k}^{\alpha}(f) \lambda_{r, k}^{\alpha}(x), \\
S_{n}^{\alpha}\left(f^{(r)}, x\right)=\sum_{k=0}^{n} c_{r, k}^{\alpha}\left(f^{(r)}\right) \lambda_{k}^{\alpha}(x) .
\end{gathered}
$$

Then

$$
\begin{gathered}
\quad\left|f(x)-S_{r, n+r}^{\alpha}(f, x)\right|= \\
=\left|\frac{1}{(r-1) !} \int_{0}^{x}(x-t)^{r-1} f^{(r)}(t) d t-\sum_{k=r}^{n+r} c_{r, k}^{\alpha}(f) \lambda_{r, k}^{\alpha}(x)\right|= \\
=\frac{1}{(r-1) !}\left|\int_{0}^{x}(x-t)^{r-1} f^{(r)}(t) d t-\sum_{k=r}^{n+r} c_{r, k}^{\alpha}(f) \int_{0}^{x}(x-t)^{r-1} \lambda_{k-r}^{\alpha}(t) d t\right|= \\
=\left|\frac{1}{(r-1) !} \int_{0}^{x}(x-t)^{r-1}\left(f^{(r)}(t)-S_{n}^{\alpha}\left(f^{(r)}, t\right)\right) d t\right| \leqslant \\
\leqslant \frac{1}{(r-1) !} \int_{0}^{x}(x-t)^{r-1}\left|f^{(r)}(t)-S_{n}^{\alpha}\left(f^{(r)}, t\right)\right| d t \leqslant \\
\leqslant \frac{1}{(r-1) !}\left(\int_{0}^{x}(x-t)^{q(r-1)} d t\right)^{1 / q}\left(\int_{0}^{x}\left|f^{(r)}(t)-S_{n}^{\alpha}\left(f^{(r)}, t\right)\right|^{p} d t\right)^{1 / p} \leqslant \\
\leqslant \frac{1}{(r-1) !}\left(\frac{A^{q(r-1)+1}}{q(r-1)+1}\right)^{1 / q}\left\|f^{(r)}-S_{n}^{\alpha}\left(f^{(r)}\right)\right\|_{L^{p}} .
\end{gathered}
$$


From equality (28) it follows that $\left\|f^{(r)}-S_{n}^{\alpha}\left(f^{(r)}\right)\right\|_{L^{p}} \rightarrow 0$ as $n \rightarrow \infty$. From this relation and (29) uniform convergence of the series (26) on $[0, A]$ to the function $f$ follows.

\section{Asymptotic properties of the functions $\lambda_{1,1+n}^{\alpha}(x)$.}

Let us study the behavior of the functions $\lambda_{1,1+n}^{\alpha}(x)$ on the segment $[0, \omega]$, where $\omega$ is a fixed positive real number.

Theorem 3. Suppose $\alpha>-1$ and $x \in[0, \omega]$. Then the following asymptotic formula holds:

$$
\begin{gathered}
\lambda_{1,1+n}^{\alpha}(x)=\sqrt{\frac{\Gamma(n+1)}{\Gamma(n+\alpha+1)}} \frac{x^{\alpha / 2+1} e^{-\frac{x}{2}}}{n+\alpha+1} \times \\
\times\left(L_{n}^{\alpha+1}(x)+\frac{x+\alpha}{2(n+\alpha+2)} L_{n}^{\alpha+2}(x)\right)+R_{n}^{\alpha}(x),
\end{gathered}
$$

where the remainder

$$
\begin{aligned}
R_{n}^{\alpha}(x)=\sqrt{\frac{\Gamma(n+1)}{\Gamma(n+\alpha+1)}} \frac{1}{4(n+\alpha+1)(n+\alpha+2)} \times \\
\quad \times \int_{0}^{x} t^{\alpha / 2}\left(t^{2}+2 \alpha t+\alpha^{2}+2 \alpha\right) e^{-\frac{t}{2}} L_{n}^{\alpha+2}(t) d t
\end{aligned}
$$

satisfies the estimate:

$$
\left|R_{n}^{\alpha}(x)\right|=O\left(\frac{1}{n}\right) .
$$

In the case $\alpha=0$, the last estimate becomes

$$
\left|R_{n}^{0}(x)\right|= \begin{cases}O\left(\frac{1}{n^{3}}\right), & 0 \leqslant x \leqslant \frac{1}{n} \\ O\left(\frac{1}{n^{7 / 4}}\right), & \frac{1}{n} \leqslant x \leqslant \omega .\end{cases}
$$

Proof. From (15), (1) and (13) it follows that

$$
\lambda_{1,1+n}^{\alpha}(x)=\int_{0}^{x} \lambda_{n}^{\alpha}(t) d t=\int_{0}^{x} t^{\alpha / 2} e^{-\frac{t}{2}} l_{n}^{\alpha}(t) d t=
$$




$$
=\sqrt{\frac{\Gamma(n+1)}{\Gamma(n+\alpha+1)}} \int_{0}^{x} t^{\alpha / 2} e^{-\frac{t}{2}} L_{n}^{\alpha}(t) d t .
$$

Further, integrating by parts and using the equality (12), we obtain:

$$
\begin{gathered}
\lambda_{1,1+n}^{\alpha}(x)=\left|\begin{array}{c}
u=\frac{e^{-\frac{t}{2}}}{t^{\alpha / 2}}, \quad d u=-\frac{e^{-\frac{t}{2}}(t+\alpha)}{2 t^{\alpha / 2+1}} \\
d v=t^{\alpha} L_{n}^{\alpha}(t) d t, \quad v=\frac{1}{n+\alpha+1} t^{\alpha+1} L_{n}^{\alpha+1}(t)
\end{array}\right|= \\
=\sqrt{\frac{\Gamma(n+1)}{\Gamma(n+\alpha+1)}\left(\frac{x^{\alpha / 2+1} e^{-\frac{x}{2}}}{n+\alpha+1} L_{n}^{\alpha+1}(x)+\right.} \\
\left.+\frac{1}{2(n+\alpha+1)} \int_{0} t^{\alpha / 2}(t+\alpha) e^{-\frac{t}{2}} L_{n}^{\alpha+1}(t) d t\right)= \\
=\left|\begin{array}{c}
u=\frac{e^{-\frac{t}{2}}(t+\alpha)}{t^{\alpha / 2+1}}, \quad d u=-\frac{e^{-\frac{t}{2}}\left(t^{2}+2 \alpha t+\alpha^{2}+2 \alpha\right)}{2 t^{\alpha / 2+2}} \\
d v=t^{\alpha+1} L_{n}^{\alpha+1}(t) d t, \quad v=\frac{1}{n+\alpha+2} t^{\alpha+2} L_{n}^{\alpha+2}(t)
\end{array}\right|= \\
=\sqrt{\frac{\Gamma(n+1)}{\Gamma(n+\alpha+1)} \frac{x^{\alpha / 2+1} e^{-\frac{x}{2}}}{n+\alpha+1}\left(L_{n}^{\alpha+1}(x)+\frac{x+\alpha}{2(n+\alpha+2)} L_{n}^{\alpha+2}(x)\right)+R_{n}^{\alpha}(x) .}
\end{gathered}
$$

Therefore, (30) holds.

Let us proceed to the estimate of the remainder $R_{n}^{\alpha}(x)$ for $0 \leqslant x \leqslant \omega$. To this end, consider the following two cases:

1 ) Let $0 \leqslant x \leqslant \frac{1}{n}$; then, from estimates (10) and (11), it follows that

$$
\begin{gathered}
\left|R_{n}^{\alpha}(x)\right| \leqslant \frac{c(\alpha)}{n^{\alpha / 2+2}} \int_{0}^{x} t^{\alpha / 2}\left(t^{2}+2|\alpha| t+\alpha^{2}+2|\alpha|\right) e^{-\frac{t}{2}}\left|L_{n}^{\alpha+2}(t)\right| d t \leqslant \\
\leqslant c(\alpha) n^{\alpha / 2}\left(\frac{1}{\alpha / 2+3} x^{\alpha / 2+3}+\frac{2|\alpha|}{\alpha / 2+2} x^{\alpha / 2+2}+\frac{\alpha^{2}+2|\alpha|}{\alpha / 2+1} x^{\alpha / 2+1}\right)=O\left(\frac{1}{n}\right) .
\end{gathered}
$$

If $\alpha=0,\left|R_{n}^{0}(x)\right|=O\left(\frac{1}{n^{3}}\right)$. 
2) Let $\frac{1}{n} \leqslant x \leqslant \omega$; then, from the formulas (7)-(9), we have:

$$
\begin{aligned}
& \left|R_{n}^{\alpha}(x)\right|=O\left(\frac{1}{n^{\alpha / 2+2}}\right) \mid \int_{0}^{1 / n} t^{\alpha / 2}\left(t^{2}+2 \alpha t+\alpha^{2}+2 \alpha\right) e^{-\frac{t}{2}} L_{n}^{\alpha+2}(t) d t+ \\
& +\int_{1 / n}^{x} t^{\alpha / 2}\left(t^{2}+2 \alpha t+\alpha^{2}+2 \alpha\right) e^{-\frac{t}{2}} L_{n}^{\alpha+2}(t) d t \mid=O\left(\frac{1}{n}\right)+ \\
& +O\left(\frac{1}{n^{\frac{\alpha}{2}+2}}\right)\left|\int_{1 / n}^{x} \frac{t^{2}+2 \alpha t+\alpha^{2}+2 \alpha}{t} N^{-\frac{\alpha}{2}-1} \frac{\Gamma(n+\alpha+3)}{n !} J_{\alpha+2}(2 \sqrt{N t}) d t\right|+ \\
& +O\left(\frac{1}{n^{\alpha / 2+2}}\right)\left|\int_{1 / n}^{x} \frac{t^{2}+2 \alpha t+\alpha^{2}+2 \alpha}{t} t^{5 / 4} O\left(n^{\alpha / 2+1 / 4}\right) d t\right| \leqslant \\
& \leqslant O\left(\frac{1}{n}\right)+O\left(\frac{1}{n^{7 / 4}}\right)+O\left(\frac{1}{n}\right)\left|\int_{1 / n}^{x} \frac{t^{2}+2 \alpha t+\alpha^{2}+2 \alpha}{t} J_{\alpha+2}(2 \sqrt{N t}) d t\right|= \\
& =O\left(\frac{1}{n}\right)+O\left(\frac{1}{n}\right) \mid \int_{1 / n}^{x} \frac{t^{2}+2 \alpha t+\alpha^{2}+2 \alpha}{t} \times \\
& \times\left[\sqrt{\frac{1}{\pi \sqrt{N t}}} \cos \left(2 \sqrt{N t}-\frac{(2 \alpha+5) \pi}{4}\right)+O\left(\frac{1}{(N t)^{3 / 4}}\right)\right] d t \mid \leqslant O\left(\frac{1}{n}\right)+ \\
& +O\left(\frac{1}{n^{5 / 4}}\right)\left|\int_{1 / n}^{x} \frac{t^{2}+2 \alpha t+\alpha^{2}+2 \alpha}{t^{5 / 4}} \cos \left(2 \sqrt{N t}-\frac{(2 \alpha+5) \pi}{4}\right) d t\right| \leqslant \\
& \leqslant O\left(\frac{1}{n}\right)+O\left(\frac{1}{n^{5 / 4}}\right) \int_{\sqrt{N / n}}^{\sqrt{N x}}\left|\frac{y^{4}+2 \alpha N y^{2}+\left(\alpha^{2}+2 \alpha\right) N^{2}}{N^{7 / 4} y^{3 / 2}}\right| d y=O\left(\frac{1}{n}\right) \text {. } \\
& \text { If } \alpha=0 \text {, then }\left|R_{n}^{0}(x)\right|=O\left(\frac{1}{n^{7 / 4}}\right) \text {. }
\end{aligned}
$$

Further, from Theorem 3 and estimates (10), (11), the following assertion is immediately deduced: 
Corollary 1 . The following estimates hold:

$$
\left|\lambda_{1, n}^{\alpha}(x)\right| \leqslant c \begin{cases}\frac{1}{n}, & 0 \leqslant x \leqslant \frac{1}{\theta_{n}}, \\ \frac{1}{n^{3 / 4}}, & \frac{1}{\theta_{n}}<x \leqslant \omega .\end{cases}
$$

Acknowledgment. The author thanks the anonymous reviewers for their valuable comments and suggestions. They contributed much to improvement of the manuscript.

This work was written with the support of the Russian Foundation for Basic Research (grant 18-31-00477 mol_a)

\section{References}

[1] Askey R., Wainger S. Mean convergence of expansions in Laguerre and Hermite series. Amer. J. Math., 1965, vol. 87, pp. 698-708. DOI: https://doi.org/10.2307/2373069.

[2] Bateman H, Erdeyi A. Higher transcendental functions. Vol. 2. McGrawHill, New York-Toronto-London, 1953.

[3] Fikhtengolts G. M. Kurs differentsial'nogo i integral'nogo ischisleniya (Course of Differential and Integral Calculus). Moscow: Fizmatlit, 2001, Vol. 2. (in Russian).

[4] Muckenhoupt B. Mean Convergence of Hermite and Laguerre Series. II. Transactions of the American Mathematical Society, 1970, 147:2, pp. 433-460. DOI: https://doi.org/10.2307/1995205.

[5] Sharapudinov I. I. Sobolev-orthogonal systems of functions associated with an orthogonal system. Izvestiya: Mathematics, 2018, 82:1, pp. 212-244. DOI: https://doi.org/10.1070/IM8536.

[6] Sharapudinov I. I., Magomed-Kasumov M. G. On Representation of a solution to the Cauchy Problem by a Fourier Series in Sobolev-Orthogonal Polynomials Generated by Laguerre Polynomials. Differ. Equations, 2018, 54:1, pp. 49-66. DOI: https://doi.org/10.1134/S0012266118010068.

[7] Sharapudinov I.I., Sharapudinov T.I. Polynomials orthogonal in the Sobolev sense, generated by Chebyshev polynomials orthogonal on a mesh. Russ. Math., 2017, 61:59, pp. 59-70. DOI: https://doi.org/10.3103/ S1066369X17080072.

[8] Sharapudinov I. I. Approximation properties of Fourier series of Sobolev orthogonal polynomials with Jacobi weight and discrete masses. Math. Notes, 2017, 101:718, pp. 718-734. DOI: https://doi.org/10.1134/ S0001434617030300. 
[9] Sharapudinov I. I., Gadzhieva Z. D., Gadzhimirzaev R. M. Systems of functions orthogonal with respect to scalar products of Sobolev type with discrete masses, generated by classical orthogonal systems. Daghestan Electronic Mathematical Reports, 2016, issue 6, pp. 31-60. (in Russian). DOI: https://doi.org/10.31029/demr.6.3.

[10] Sharapudinov I. I., Gadzhieva Z. D., Gadzhimirzaev R. M. Difference equations and Sobolev orthogonal polynomials, generated by Meixner polynomials. Vladikavkaz Math. J., 2017, 19:2, pp. 58-72. (in Russian). DOI: https://doi.org/10.23671/VNC.2017.2.6509.

[11] Sharapudinov I. I., Sharapudinov T. I. Mixed series of Jacobi and Chebyshev polynomials and their discretization. Math. Notes, 2010, 88:1, pp. 112-139. DOI: https://doi.org/10.1134/S0001434610070114

[12] Szegö G. Orthogonal Polynomials. Amer. Math. Soc. Colloq. Publ, 23, Amer. Math. Soc., Providence R.I. 1975. Fourth Edition.

Received November 02, 2018.

In revised form, February 03, 2019.

Accepted February 04, 2019.

Published online February 11, 2019.

Dagestan Scientific Center of RAS

45, M.Gadzhieva st., Makhachkala, 367025, Russia

E-mail: ramis3004@gmail.com 